\title{
Delaminid for multidrug-resistant pulmonary tuberculosis
}

Delaminid (OPC-67683) is an investigational drug for tuberculosis from a new class (nitro-dihydro-imidazooxazole derivatives) that inhibits synthesis of mycolic acid. This multicentre phase 2 randomised clinical trial evaluated the efficacy and safety of delaminid at two doses (100 mg bd, $200 \mathrm{mg}$ bd) versus placebo administered for 8 weeks in combination with a standardised background regimen for multidrug-resistant pulmonary tuberculosis.

Delaminid increased the proportion achieving sputum culture conversion at 2 months (45\% and $42 \%$ at $100 \mathrm{mg}$ and $200 \mathrm{mg}$, respectively) compared with placebo (30\%). Time to culture conversion was also significantly shorter with delaminid.

In general, delaminid had an acceptable safety profile, but the one concerning finding was that prolongation of QT interval on ECG was more common in both delaminid groups (10\% and $13 \%$ for $100 \mathrm{mg}$ and $200 \mathrm{mg}$, respectively) compared with placebo (4\%). The episodes of prolonged QT interval were not associated with any clinical events. Further surveillance for cardiotoxicity will be essential as development moves forward.

Delaminid is one of a number of drugs that now offers hope for improved treatment of drug-resistant tuberculosis. It should be noted that this trial only included four HIV-infected participants and excluded those with CD 4 cell count $<350$ cells/ $\mu$ l and those on antiretroviral therapy. A phase 3 trial is already underway, which will, in certain sites, include HIV co-infected participants, and which will report on longer-term outcomes. The use of delaminid alongside other investigational agents in novel regimens is also an area of particular interest for future research.

- Gler MT, Skripconoka V, Sanchez-Garavito E, et al. Delaminid for multidrug-resistant pulmonary tuberculosis. N Engl J Med 2012;366:2151-60.

\section{Richard J Lessells}

Correspondence to Dr Richard J Lessells, Department of Clinical Research, London School of Hygiene and Tropical Medicine, Keppel Street, London WC1E 7HT, UK; richard.lessells@|shtm.ac.uk

Competing interests None.

Provenance and peer review Not commissioned; internally peer reviewed.

To cite Lessells RJ. Thorax 2013;68:730.

Published Online First 24 August 2012

Thorax 2013;68:730. doi:10.1136/thoraxjnl-2012-202520 